\title{
Comet 66P/du Toit: not a near-Earth main belt comet
}

\author{
Bin Yang (杨涁) ${ }^{1}$, Emmanuël Jehin ${ }^{2}$, Francisco J. Pozuelos ${ }^{2,4}$, Youssef Moulane ${ }^{1,2,3}$, Yoshiharu Shinnaka ${ }^{5}$, \\ Cyrielle Opitom ${ }^{1}$, Henry H. Hsieh ${ }^{6}$, Damien Hutsemékers ${ }^{2}$, and Jean Manfroid ${ }^{2}$ \\ ${ }^{1}$ European Southern Observatory, Alonso de Còrdova 3107, Vitacura, Casilla 19001, Santiago, Chile \\ e-mail: byang@eso.org \\ ${ }^{2}$ Space sciences, Technologies \& Astrophysics Research (STAR) Institute, Université de Liège, 4000 Liège, Belgium \\ ${ }^{3}$ Oukaimeden Observatory, High Energy Physics and Astrophysics Laboratory, Cadi Ayyad University, Marrakech, Morocco \\ ${ }^{4}$ EXOTIC Lab, UR Astrobiology, AGO Department, University of Liège, 4000 Liège, Belgium \\ ${ }^{5}$ Laboratory of Infrared High-resolution Spectroscopy, Koyama Astronomical Observatory, Kyoto Sangyo University, Motoyama, \\ Kamigamo, Kita-ku, Kyoto 603-8555, Japan \\ ${ }^{6}$ Planetary Science Institute, 1700 East Fort Lowell, Suite 106, Tucson, AZ 85719, USA
}

Received 6 August 2019 / Accepted 14 September 2019

\begin{abstract}
Context. Main belt comets (MBCs) are a peculiar class of volatile-containing objects with comet-like morphology and asteroid-like orbits. However, MBCs are challenging targets to study remotely due to their small sizes and the relatively large distance they are from the Sun and the Earth. Recently, a number of weakly active short-period comets have been identified that might originate in the asteroid main belt. Among all of the known candidates, comet 66P/du Toit has been suggested to have one of the highest probabilities of coming from the main belt.

Aims. The main goal of this study is to investigate the physical properties of $66 \mathrm{P}$ via spectroscopic and imaging observations to constrain its formation conditions. In particular, the isotopic abundance ratio and the ortho-to-para ratio (OPR) of gaseous species can be derived via high-resolution spectroscopy, which is sensitive to the formation temperature of the nucleus.

Methods. We obtained medium and high-resolution spectra of 66P from 300-2500 $\mathrm{nm}$ with the X-shooter and the UVES instruments at the Very Large Telescope in July 2018. We also obtained a series of narrow-band images of 66P to monitor the gas and dust activity between May and July 2018 with TRAPPIST-South. In addition, we applied a dust model to characterize the dust coma of 66P and performed dynamical simulations to study the orbital evolution of 66P.

Results. We derive the OPR of ammonia $\left(\mathrm{NH}_{3}\right)$ in $66 \mathrm{P}$ to be $1.08 \pm 0.06$, which corresponds to a nuclear spin temperature of $\sim 34 \mathrm{~K}$. We compute the production rates of $\mathrm{OH}, \mathrm{NH}, \mathrm{CN}, \mathrm{C}_{3}$, and $\mathrm{C}_{2}$ radicals and measure the dust proxy, $A f \rho$. The dust analysis reveals that the coma can be best-fit with an anisotropic model and the peak dust production rate is about $55 \mathrm{~kg} \mathrm{~s}^{-1}$ at the perihelion distance of $1.29 \mathrm{au}$. Dynamical simulations show that $66 \mathrm{P}$ is moderately asteroidal with the capture time, $t_{\text {cap }} \sim 10^{4} \mathrm{yr}$.

Conclusions. Our observations demonstrate that the measured physical properties of $66 \mathrm{P}$ are consistent with typical short-period comets and differ significantly from other MBCs. Therefore, 66P is unlikely to have a main belt origin.
\end{abstract}

Key words. comets: general - comets: individual: 66P/du Toit - methods: observational - methods: numerical

\section{Introduction}

Water is essential for life and it is an important tracer of the formation and evolution processes in planetary systems. In addition, the distribution of water and volatiles in our solar system is a primary determinant of habitability. Recent space mission results and ground-based observations show that water is prevalent throughout the solar system. This includes many previously unexpected locations, such as the asteroid main belt, where most known asteroids reside in the region between the orbits of Mars and Jupiter. The so called main belt comets (MBCs) are a peculiar population with a comet-like morphology and asteroid-like orbits, which most likely contain buried water ice (Hsieh \& Jewitt 2006). The MBCs, thus, are particularly important in relation to the history of water and other major volatiles. They are appealing targets for future space missions since in situ sampling could be possible with similar instruments as those on Rosetta (Snodgrass et al. 2017a). However, MBCs are observationally challenging targets not only because they are small (merely a few $\mathrm{km}$ across), but also because they exhibit very low activity. Even with the most powerful telescopes (i.e., Herschel, Keck, VLT,
GEMINI, and GTC), all of the previous attempts failed to detect any evidence of gaseous products of sublimation (Hsieh et al. 2011; de Val-Borro et al. 2012; Licandro et al. 2013; Snodgrass et al. 2017b). These studies demonstrate that spectroscopic detection of gas at main-belt distances is extremely difficult due to the rapidly declining water sublimation rates from 2 to 3 au (Jewitt et al. 2015).

Recently, Fernández \& Sosa (2015) identified a number of near-Earth short-period comets or Jupiter family comets (JFCs) that are more dynamically stable and exhibit weaker activity than other JFCs. Furthermore, they suggest that these objects might originate in the main asteroid belt. This possibility is also supported by Hsieh \& Haghighipour (2016), who find that main-belt asteroids can indeed attain JFC-type orbits under certain circumstances. We call these anomalous objects "near-Earth MBC candidates," or NEMBC candidates. Among all of the known NEMBC candidates, comet $66 \mathrm{P}$ is defined by the authors as highly asteroidal (while still satisfying the dynamical requirements to be considered a short-period comet), meaning that there is a high probability that this object comes from the main belt (Fernández \& Sosa 2015). Thus, 66P is an attractive target 
Table 1. Journal of VLT and TRAPPIST observations, and derived $\mathrm{OH}, \mathrm{CN}, \mathrm{C}_{2}$, and $\mathrm{C}_{3}$ production rates and $A\left(\theta=0^{\circ}\right) f \rho$ parameter of comet $66 \mathrm{P}$.

\begin{tabular}{|c|c|c|c|c|c|c|c|c|c|c|c|}
\hline \multirow[t]{2}{*}{ UT date } & \multirow[t]{2}{*}{ Tel } & \multirow{2}{*}{$\begin{array}{c}r_{\mathrm{h}} \\
(\mathrm{au})\end{array}$} & \multirow{2}{*}{$\begin{array}{c}\Delta \\
(\mathrm{au}) \\
\end{array}$} & \multicolumn{4}{|c|}{ Production rates $\left(10^{24}\right.$ molecules $\left.\mathrm{s}^{-1}\right)$} & \multicolumn{4}{|c|}{$A\left(\theta=0^{\circ}\right) f \rho(\mathrm{cm})$} \\
\hline & & & & $\mathrm{Q}(\mathrm{OH})$ & $\mathrm{Q}(\mathrm{CN})$ & $\mathrm{Q}\left(\mathrm{C}_{2}\right)$ & $\mathrm{Q}\left(\mathrm{C}_{3}\right)$ & $\mathrm{BC}$ & $\mathrm{RC}$ & $\mathrm{Rc}$ & Ic \\
\hline 2018-05-06.4 & TRAPPIST & 1.30 & 0.90 & & $5.67 \pm 0.55$ & $4.65 \pm 0.68$ & & & & & \\
\hline $2018-05-16.4$ & TRAPPIST & 1.29 & 0.90 & $2880 \pm 297$ & $8.59 \pm 0.53$ & $8.63 \pm 0.70$ & $2.67 \pm 0.20$ & $82.6 \pm 6.3$ & $108.0 \pm 7.7$ & $102.4 \pm 6.3$ & $98.6 \pm 8.6$ \\
\hline $2018-05-23.4$ & TRAPPIST & 1.29 & 0.90 & $2530 \pm 298$ & $8.00 \pm 0.53$ & $8.22 \pm 0.64$ & $2.43 \pm 0.22$ & $75.6 \pm 5.7$ & & & \\
\hline $2018-05-26.4$ & TRAPPIST & 1.29 & 0.90 & & $7.60 \pm 0.52$ & $7.78 \pm 0.64$ & $2.20 \pm 0.21$ & $70.8 \pm 6.0$ & $97.7 \pm 6.1$ & $76.7 \pm 6.9$ & $88.0 \pm 8.6$ \\
\hline 2018-06-17.4 & TRAPPIST & 1.34 & 0.91 & & $6.23 \pm 0.56$ & $3.40 \pm 0.67$ & $1.26 \pm 0.23$ & & & $46.9 \pm 6.8$ & $55.2 \pm 7.4$ \\
\hline $2018-06-28.4$ & TRAPPIST & 1.39 & 0.92 & & $3.60 \pm 0.56$ & $3.85 \pm 0.68$ & & & & & \\
\hline $2018-07-02.3$ & VLT/UVES & 1.42 & 0.92 & & & & & & & & \\
\hline 2018-07-08.4 & VLT/XSH & 1.45 & 0.92 & $891 \pm 280$ & $2.15 \pm 0.20$ & $2.07 \pm 0.20$ & $1.92 \pm 0.20$ & & & & \\
\hline $2018-07-13.4$ & TRAPPIST & 1.48 & 0.92 & & $1.65 \pm 0.51$ & $1.40 \pm 0.62$ & & & & & \\
\hline $2018-07-14.4$ & VLT/XSH & 1.49 & 0.92 & $541 \pm 230$ & $1.53 \pm 0.20$ & $1.78 \pm 0.20$ & $1.26 \pm 0.20$ & & & & \\
\hline
\end{tabular}

Notes. $r_{\mathrm{h}}$ and $\Delta$ are the heliocentric and geocentric distances, respectively. The $A(0) f \rho$ values are printed at $5000 \mathrm{~km}$ from the nucleus and they are corrected for the phase angle effect. The perihelion of 66P was on May 20, 2018 when the comet was at 1.28 au from the Sun and at 0.90 au from Earth.

because it may represent a sample of MBCs that is more accessible than their main belt counterparts. If their main belt origin is confirmed, the newly identified NEMBC candidates (such as $66 \mathrm{P}$ ) represent the best sample with which we can hope to take a close look at MBCs and investigate their composition in details other than a spacecraft visit. In turn, studying NEMBCs will give us a window to estimate MBC compositions and test terrestrial water origin models. If 66P forms in the asteroid belt, we would expect its physical properties, such as composition, isotopic ratio, and water abundance, to be distinct from those of typical JFCs. These properties can be derived through spectroscopic and photometric observations. In Sect. 2, we present observations performed with the ESO's Very Large Telescope (VLT) and the TRAPPIST-South telescope. In Sect. 3, we present the observational results as well as the results of our dust models. In Sect. 4, we revisit the dynamical evolution of 66P, following the approach in Fernández \& Sosa (2015) but by using the latest orbital parameters. Lastly, in Sect. 5, we summarize all the observational and theoretical simulation results and present our conclusion on the origin of $66 \mathrm{P}$.

\section{Observations and data reduction}

\subsection{X-shooter observations}

The chemical composition of comets can be investigated by observing gas emission bands in the UV and visible, and potentially by observing absorption features in the near-infrared (NIR) continuum due to ice grains or various minerals in grains. $\mathrm{X}$-shooter allows all possibilities to be explored in one exposure. Notably, the $\mathrm{OH}$ emission band at $308 \mathrm{~nm}$ can be observed in the bluest order of the UVB arm, which in turn is used to estimate the total water production rate. Traditionally, other gas production rates are compared to the water production rate as a reference. We were allotted $2.7 \mathrm{~h}$ of director discretionary time to observe $66 \mathrm{P}$ with $\mathrm{X}$-shooter. The two sets of observations took place in service mode a few days apart in July 2018. Details on the observing geometry are given in Table 1 . We used slit widths $1.3^{\prime \prime}, 1.2^{\prime \prime}$, and $1.2^{\prime \prime}$ in the UVB, VIS, and NIR arms, respectively. The spatial scale covers from the optocenter up to $3700 \mathrm{~km}$ in both directions. The overall wavelength range runs from 300 to $2500 \mathrm{~nm}$ with the spectral resolution of 4100 in the UVB arm, 6500 in the VIS arm, and 4300 in the NIR. We also observed the solar analog star SA93-101 immediately after the comet on both nights.
The X-shooter data is reduced using the Reflex environment (Freudling et al. 2013) based on the ESO XSHOOTER pipeline (Modigliani et al. 2010). We used the Reflex pipeline to obtain the two dimensional order-merged and wavelength-calibrated spectra, while the one-dimensional spectra of 66P were extracted using self-developed IDL routines.

\subsection{UVES observations}

Observations of comet 66P were carried out in service mode with the Ultraviolet and Visual Echelle Spectrograph (UVES) mounted on the $8.2 \mathrm{~m} \mathrm{UT2}$ telescope of the European Southern Observatory. Using director discretionary time, the total $7200 \mathrm{~s}$ of science exposure was divided into two exposures of $3600 \mathrm{~s}$ each on July 2, 2018. We used the atmospheric dispersion corrector and the UVES standard setting DIC\#1 346 + 580, which roughly covers from 300 to $388 \mathrm{~nm}$ on the blue CCD and from 476 to $684 \mathrm{~nm}$ on the red mosaic CCD. We used a $0.5 \times 1.0^{\prime \prime}$ slit, providing a resolving power $R \sim 80000$.

The raw spectral data were reduced using the UVES Common Pipeline Library (CPL) data reduction pipeline (Ballester et al. 2000), and modified to accurately merge individual orders into a two-dimensional spectrum. Subsequently, the echelle package of the IRAF software was used to calibrate the spectra and to extract one-dimensional spectra. In turn, the cosmic rays were removed and comet spectra were rebinned and corrected for the velocity of the comet. Lastly, the continuum component, including the sunlight reflected by cometary dust grains and the telluric absorption features, was removed.

\subsection{TRAPPIST observations}

We observed comet 66P around its perihelion (1.29 au) with the TRAPPIST-South $60-\mathrm{cm}$ robotic telescope at the La Silla observatory (Jehin et al. 2011). We used the HB narrow band filters (Farnham et al. 2000), isolating the emission bands of $\mathrm{OH}[310 \mathrm{~nm}], \mathrm{CN}[385 \mathrm{~nm}], \mathrm{C}_{3}[405 \mathrm{~nm}]$, and $\mathrm{C}_{2}[515 \mathrm{~nm}]$ as well as emission free continuum BC[445 nm] and RC[715 nm] regions. We also took images with broad band $B, V, R_{c}$, and $I_{c}$ Johnson-Cousin filters. Since its discovery in 1944, 66P showed a highly variable appearance during its previous perihelion passages with its visual brightness varying between 10th and 20th magnitude. We monitored the activity of this comet for two months, from May 6 to July 13, 2018. During this period, we detected the strong $\mathrm{CN}, \mathrm{C}_{2}$, and $\mathrm{C}_{3}$ emissions on most of the 
nights while $\mathrm{OH}$ was only detected two times with a low signalto-noise ratio $(\mathrm{S} / \mathrm{N})$. $\mathrm{NH}$ was not detected due to the weak activity of this comet.

Standard procedures were used to calibrate the data by the creation of master bias, flat, and dark frames. The bias and dark subtraction, as well as the flat-field correction were done. The absolute flux calibration was made using standard stars observed during the same period (Farnham et al. 2000). We removed the sky background using the procedure developed in previous papers (Opitom et al. 2015a,b, 2016; Moulane et al. 2018). We derived median radial brightness profiles for the gas and dust images. We removed the dust contamination from the gas radial profiles using images of the comet taken in the $\mathrm{BC}$ filter (Farnham et al. 2000). In order to derive the production rates, we converted the flux of the different gas species $\left(\mathrm{OH}, \mathrm{CN}, \mathrm{C}_{3}\right.$, and $\mathrm{C}_{2}$ ) to column densities and estimated their profiles with the Haser model (Haser 1957).

For dust modeling, we used the broad band $R$ JonsonCousins filters. In order to improve the $\mathrm{S} / \mathrm{N}$, the comet was imaged several times each night using integration times in the range $60-120 \mathrm{~s}$. The individual images were flat-fielded and bias subtracted using standard techniques, then a median stack was obtained from the available images. The flux calibration was done using the USNO-B1.0 star catalog (Monet et al. 2003). Images were calibrated in mag $\operatorname{arcsec}^{-2}$, and then converted to solar disk units (SDUs). The USNO-B1.0 star catalog provides a photometric accuracy of 0.3 mag and 0. " 2 of astrometric precision, which are sufficient for our modeling purposes.

\section{Analysis and results}

\subsection{Medium resolution spectroscopy with $X$-shooter}

X-shooter/UVB spectra of comet 66P acquired in July 2018 are shown in Fig. 1. The first X-shooter observation was made about 1.5 months post-perihelion, the comet was still quite active as shown by the TRAPPIST observations. Several common cometary species were detected, such as $\mathrm{OH}, \mathrm{CN}, \mathrm{C}_{2}, \mathrm{C}_{3}$, and $\mathrm{NH}$. We derived production rates of these species using a simple Haser model (Haser 1957), following the method described in Hsieh et al. (2011). The second X-shooter observation was made a week after the first one, the comet was noticeably much fainter when further away from the Sun. Nevertheless, all the major cometary species were detected the second time. Our results of the gas production rates based on the $\mathrm{X}$-shooter observations are listed in Table 1, which are consistent with the values derived from the TRAPPIST data.

The merged and normalized 66P spectrum in comparison to the major asteroid spectral classes (DeMeo et al. 2009) is shown in Fig. 2. The relative reflectance spectrum of 66P appears featureless with no sign of the presence of water ice or hydrated minerals. The comet continuum has a reddish spectral slope, which is similar to the mean spectral slope of the D-type asteroids and significantly deviates from the mean spectral slope of the C-type asteroids. In contrast, the reflectance spectra of the majority of the known MBCs show neutral to slightly bluish slopes, which resemble the C-type spectra.

\subsection{High resolution spectroscopy with UVES}

Due to the close proximity of $66 \mathrm{P}$ at the time of the observations, we were able to measure the abundance ratio of the nuclear spin isomers of $\mathrm{NH}_{2}$, namely the ortho-to-para ratio (OPR), shown in Fig. 3. In adopting the method described in Kawakita et al. (2001), we further derived the OPR of $\mathrm{NH}_{3}$ using the

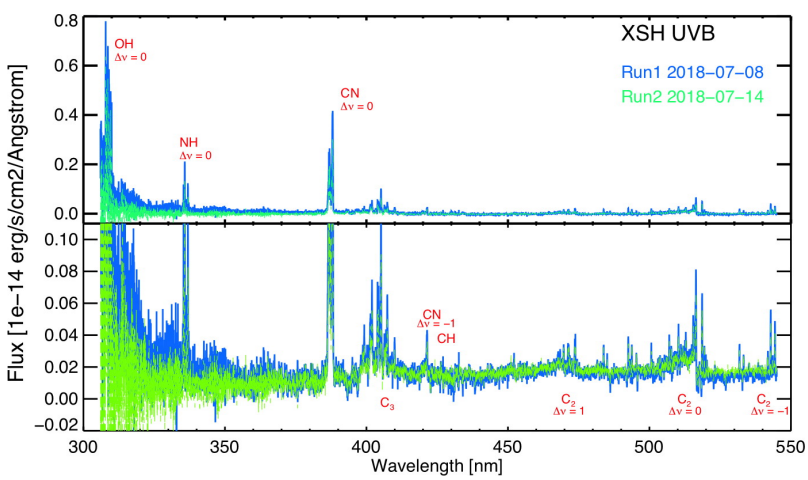

Fig. 1. Flux-calibrated spectra of comet $66 \mathrm{P}$ acquired with $\mathrm{X}$-shooter on July 8 and 14 . The strongest cometary emission features in the UV and optical range $\left(\mathrm{OH}, \mathrm{NH}, \mathrm{CN}, \mathrm{C}_{2}\right.$, and $\left.\mathrm{C}_{3}\right)$ are marked.

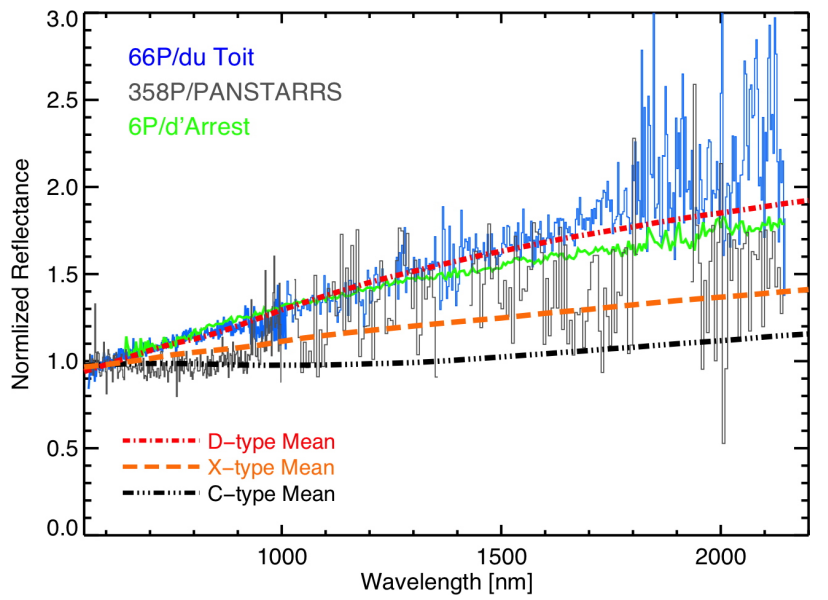

Fig. 2. Combined VIS and NIR reflectance spectrum of 66P is shown as the blue line. Some emission-like features in the NIR part are due to imperfect removal of the telluric absorption bands. The spectrum of the MBC, 358P, was taken with X-shooter and is from Snodgrass et al. (2017b). Spectrum of the JFC, 6P/d'Arrest, was observed with the IRTF telescope and is from Yang (2009). The comet continuum shows a red spectral slope, which is similar to the D-type (shown as the red dashed line) and significantly deviates from the C-type (shown as the black dashed line). Three asteroid spectral classes are taken from DeMeo et al. (2009).

high-dispersion spectrum of $\mathrm{NH}_{2}$, as seen in Table 2. Given that $\mathrm{NH}_{3}$ is directly incorporated into the nucleus, the nuclear spin temperature of ammonia sets strong constraints on the formation environment of the comet. Our results are shown in Fig. 4, where the $\mathrm{NH}_{3}$ OPR value of $66 \mathrm{P}$ is comparable to those of other comets.

We attempted to measure the nitrogen and carbon isotopic ratios from the $\mathrm{CN}$ violet $(0,0)$ band following the method of Manfroid et al. (2009). However, the observing conditions were not optimal for isotopic measurement: the comet was close to a nearly full moon and there were some cirrus clouds; moreover, the rate of outgassing of 66P was low and the total integration time for the UVES observation was not long enough to reach a desired $\mathrm{S} / \mathrm{N}$. As such, we were not able to derive either the ${ }^{12} \mathrm{C} /{ }^{13} \mathrm{C}$ or the ${ }^{14} \mathrm{~N} /{ }^{15} \mathrm{~N}$ ratio.

\subsection{Narrow and broad band photometry with TRAPPIST-South}

The $\mathrm{OH}, \mathrm{CN}, \mathrm{C}_{2}$, and $\mathrm{C}_{3}$ production rates as well as the $A f \rho$ values (A'Hearn et al. 1984) from TRAPPIST-South are given 


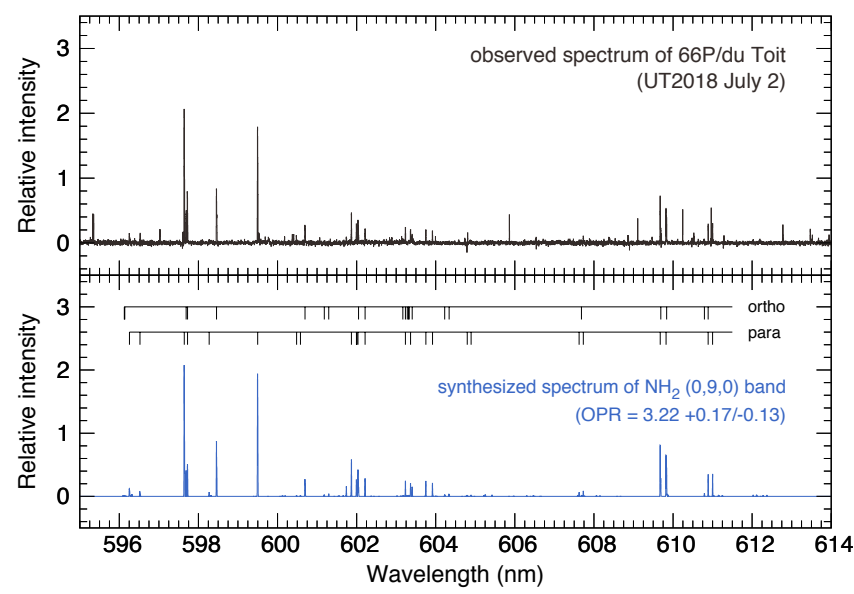

Fig. 3. Comparison between observed and modeled spectrum of $\mathrm{NH}_{2}$.

Table 2. Derived $\mathrm{NH}_{2}$ and $\mathrm{NH}_{3}$ OPRs and nuclear spin temperature.

\begin{tabular}{lccc}
\hline \hline $\mathrm{NH}_{2}$ band & $\mathrm{NH}_{2} \mathrm{OPR}$ & $\mathrm{NH}_{3} \mathrm{OPR}$ & $T_{\text {spin }}$ \\
\hline$(0,7,0)$ & $3.18^{+0.17} /-0.13$ & $1.09^{+0.09} /-0.07$ & $32^{+12} /-5$ \\
$(0,8,0)$ & $3.00^{+0.28} /-0.34$ & $1.00^{+0.14} /-0.17$ & $>21(3-\sigma)$ \\
$(0,9,0)$ & $3.22^{+0.21} /-0.29$ & $1.11^{+0.11 /-0.15}$ & $31^{+27} /-7$ \\
Mean & $3.16^{+0.12} /-0.11$ & $1.08 \pm 0.06$ & $34^{+12} /-5$ \\
\hline
\end{tabular}

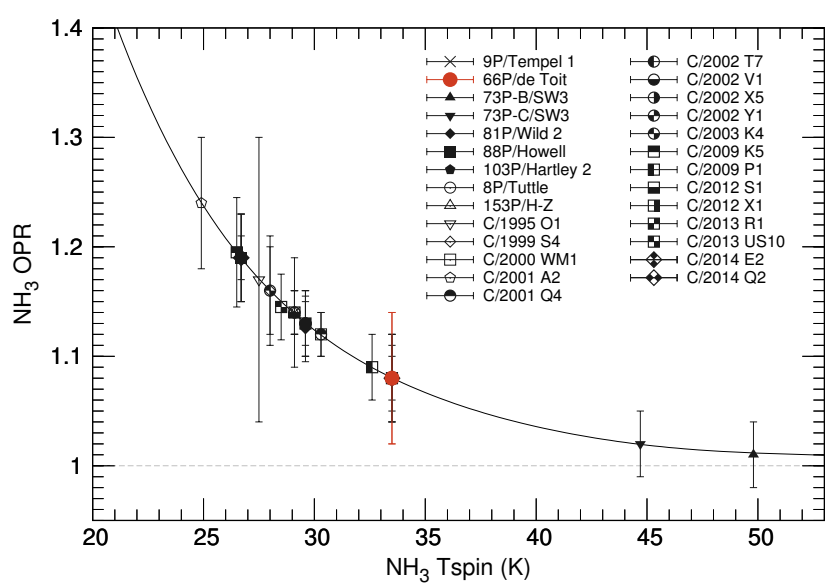

Fig. 4. $\mathrm{NH}_{3}$ ortho-to-para ratios (OPRs) of comets converted from those of $\mathrm{NH}_{2}$ and corresponding spin temperatures of $\mathrm{NH}_{3}$. OPRs of other comets besides 66P are taken from Shinnaka et al. (2016). The horizontal dashed line indicates the nuclear-spin statistical weights ratio of ammonia (1.0).

in Table 1. The activity of $66 \mathrm{P}$ did not change much around perihelion (1.30-1.29 au), but it started to decrease at $1.37 \mathrm{au}$. We estimate a water production rate of about $(3.24 \pm 0.17) \times$ $10^{27}$ molecules $^{-1}$ around perihelion, which was derived from the mean values of $\mathrm{Q}(\mathrm{OH})$ using $\mathrm{Q}\left(\mathrm{H}_{2} \mathrm{O}\right)=1.361 \times r_{\mathrm{h}}^{-0.5} \times \mathrm{Q}(\mathrm{OH})$, given in Cochran \& Schleicher (1993).

We computed the mean production rate ratios of $\mathrm{CN} / \mathrm{OH}$, $\mathrm{C}_{2} / \mathrm{OH}$, and $\mathrm{C}_{2} / \mathrm{CN}$ as well as the $A f \rho /$ gas ratios, such as $A(\theta=0) f \rho / \mathrm{OH}$ and $A(\theta=0) f \rho / \mathrm{CN}$. Table 3 summarizes these ratios and compares them to the typical values of comets based on the narrowband photometry survey of over 100 comets, given in Schleicher (2008). Our results show that the mixing ratio of various carbon-chain molecules of $66 \mathrm{P}$ are compatible with the composition of typical comets.
Table 3. Mean production rate ratios and $A f \rho /$ gas ratios for comet $66 \mathrm{P}$ compared to mean values of carbon-chain typical comets presented in Schleicher (2008).

\begin{tabular}{lcc}
\hline \hline & \multicolumn{2}{c}{ Log production rate ratio } \\
\cline { 2 - 3 } Species & $66 \mathrm{P} / \mathrm{du}$ Toit & Schleicher $(2008)$ \\
\hline $\mathrm{C}_{2} / \mathrm{CN}$ & $0.04 \pm 0.03$ & 0.10 \\
$\mathrm{C}_{2} / \mathrm{OH}$ & $-2.69 \pm 0.04$ & -2.46 \\
$\mathrm{C}_{3} / \mathrm{OH}$ & $-3.10 \pm 0.04$ & -3.12 \\
$\mathrm{CN} / \mathrm{OH}$ & $-2.66 \pm 0.03$ & -2.55 \\
$A(0) f \rho / \mathrm{OH}$ & $-25.55 \pm 0.03$ & $-25.84 \pm 0.40^{(a)}$ \\
$A(0) f \rho / \mathrm{CN}$ & $-22.87 \pm 0.03$ & - \\
\hline
\end{tabular}

Notes. The $A(0) f \rho /$ gas ratio has units of $\mathrm{cm} \mathrm{s}$ molecules ${ }^{-1}$. $^{(a)}$ This value is from A'Hearn et al. (1995).

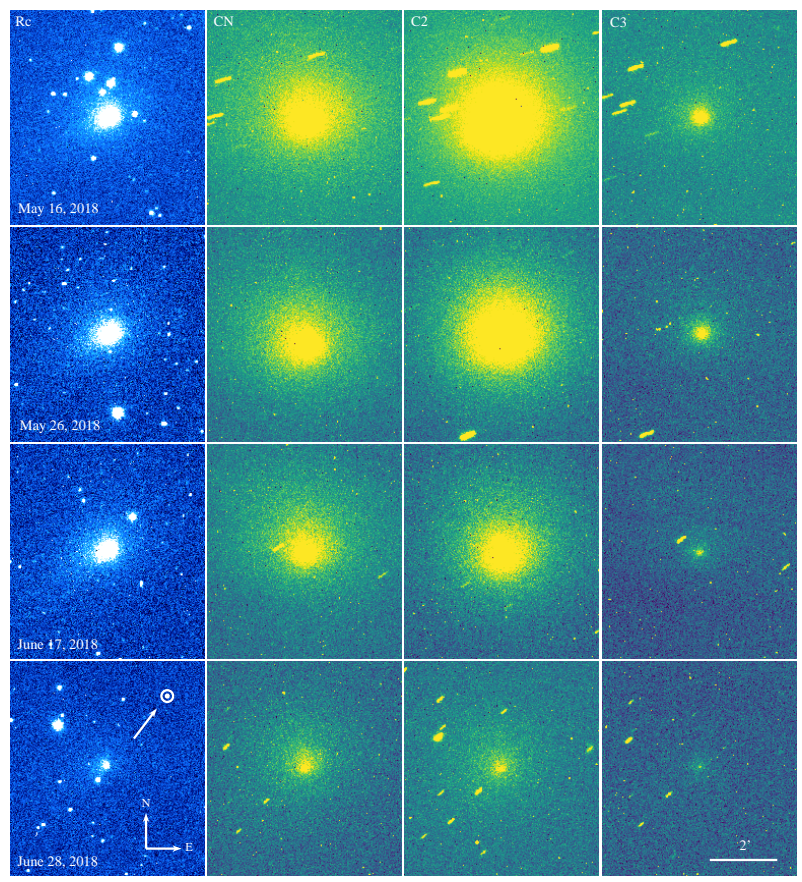

Fig. 5. Evolution of 66P coma morphology in Rc, $\mathrm{CN}, \mathrm{C}_{2}$, and $\mathrm{C}_{3}$ filter images of TRAPPIST. The orientation and scale are given at the bottom of the images.

We searched for morphological features in the coma, but no jet was detected in the narrow-band or broad-band images due to the low activity of the comet. Figure 5 shows the CN coma evolution over time. It was nearly spherically symmetric throughout the monitoring window. 66P reached perihelion on May 20, 2018 when the coma was the brightest. Since then, the comet faded gradually and steadily.

\subsection{Dust environment evolution}

In order to constrain the physical properties of 66P's dust coma using the TRAPPIST observations, we adopted the Monte Carlo dust tail code described in Moreno et al. (2012), which has been used previously for several active asteroids and comets (e.g., Moreno et al. 2014, 2016b). The code produces synthetic images that can be compared directly with actual observations. These images are generated by adding the contribution to the brightness of each dust-like particle to mimic the cometary tail. A number of assumptions regarding the physical parameters of the 
dust must be made to make the problem more tractable. Based on in situ measurements of the dust coma of 67P from Rosetta's Optical, Spectroscopic, and Infrared Remote Imaging System (OSIRIS) and Grain Impact Analyzer and Dust Accumulator (GIADA), we assume the density of the dust particles and the geometric albedo as $\rho=1000 \mathrm{~kg} \mathrm{~m}^{-3}$ and $p_{v}=0.065$, respectively (Fulle et al. 2016b; Fornasier et al. 2015). The minimum size of the particles radius, $r_{\text {dmin }}$, was set to a constant value of $1 \mu \mathrm{m}$. This choice was motivated by results from the MicroImaging Dust Analysis System (MIDAS; Riedler et al. 2007) on board Rosetta. Most of the particles of 67P were found to be hierarchical agglomerates up to a few tens of microns, which consist of micron-sized sub-units (Mannel et al. 2016; Bentley et al. 2016). Furthermore, we tested minimum values of 5 and $10 \mu \mathrm{m}$, and no significant change was observed. The maximum particle radius, $r_{\mathrm{dmax}}(t)$, is considered as a time-dependent parameter up to decimeter sizes (Rotundi et al. 2015, Fulle et al. 2016a). The size distribution of the particles follows a power-law function given by $n(r) \propto r^{\delta(t)}$, where $\delta(t)$ is a time-dependent parameter that ranges from -4.2 to -2.0 (Fulle et al. 2016b, Ott et al. 2017). The terminal velocity of the particles depends on the activation mechanism involved. Since the comet $66 \mathrm{P}$ showed activity during previous perihelion passages, it is most likely that the activity is driven by ice sublimation. Therefore, we assume a canonical parameterization given by $v(t, \beta)=v_{0}(t) \times \beta^{\gamma}$, where $\gamma$ is set to 0.5 (see e.g., Whipple 1951; Della Corte et al. 2016). The term $v_{0}(t)$ is a time-dependent parameter. Besides $v_{0}(t)$, the power-law index of the particle size distribution $\delta(t)$, the maximum size of the particles, $r_{\mathrm{dmax}}(t)$, and the dust mass loss rate, $Q_{\text {dust }}(t)$, are also time-dependent. All of these time variables are determined during the modeling process, which consists of a trial-and-error procedure, where a grid of possible combinations for the dust parameters defined is explored. In order to determine the goodness of the model during the fitting process, we computed the quantity $\chi$ for every trial following Moreno et al. (2016a), looking for its minimum value.

After a long set of runs using an isotropic ejection model, we find that this model does not offer a good match for the observations. Due to the poor goodness-of-fit using the isotropic model, we then considered an anisotropic ejection pattern, where the emission of the particles is characterized by active areas on the comet's surface, and the rotational state is defined by two angles (Sekanina 1981): the obliquity of the orbital plane to the equator, $I$, and the argument of the subsolar meridian at perihelion, $\phi$. The obliquity determines the direction of the rotation, which is prograde when $0^{\circ} \leq I<90^{\circ}$ and retrograde when $90^{\circ}<I \leq 180^{\circ}$. When $0^{\circ}<\phi<180^{\circ}$, the northern pole experiences sunlight at perihelion, while the southern pole receives sunlight when $180^{\circ}<\phi<360^{\circ}$. In this context, we find that the anisotropic model provides a much better fit that consists of an ejection of particles coming from the northern hemisphere (ranging from $\left.0^{\circ}-90^{\circ}\right)$. The rotational parameters found are $I=(30 \pm 10)^{\circ}$ and $\phi=(20 \pm 5)^{\circ}$, that is, the northern hemisphere receives sunlight during the perihelion passage.

Overall, the best-fitting model suggests that the activity started $\sim 150$ days before perihelion. However, the lack of observational information pre-perihelion, means we can not unambiguously determine the starting date of the activity: models with starting dates between 130 and 200 days preperihelion yield similar results, such as $\chi(t=-200)=4.09$, $\chi(t=-150)=3.95$, and $\chi(t=-130)=4.15$. The dust production rate shows nearly symmetric behavior with respect to perihelion (see Fig. 6), where the peak of the activity is $Q_{\text {dust }} \approx 55 \mathrm{~kg} \mathrm{~s}^{-1}$ at perihelion distance, and the total dust ejected from the starting

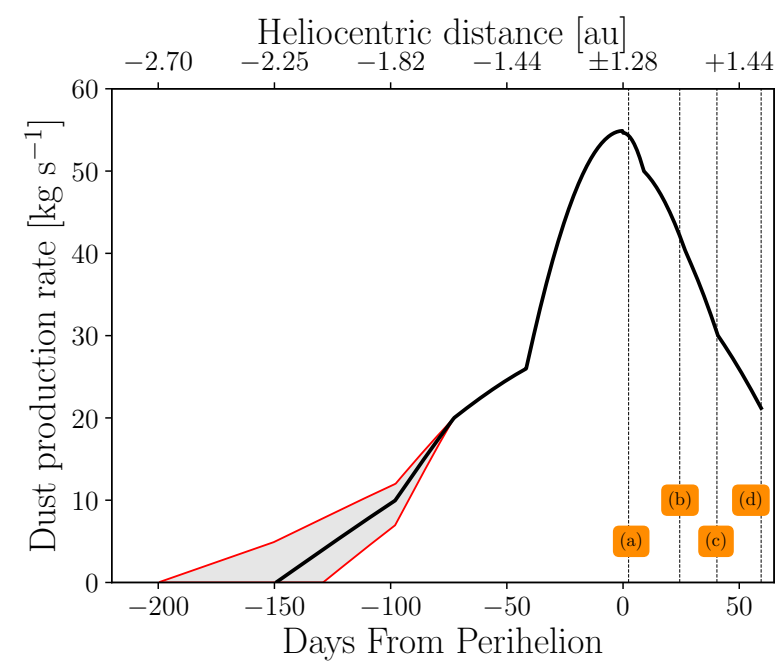

Fig. 6. Dust production rate given by best-fitting model as function of heliocentric distance (upper $x$-axis) and day relative to perihelion (lower $x$-axis). Dashed-vertical lines correspond to the observation dates presented in Fig. 8 and summarized in Table 4. The gray shaded area corresponds to different starting dates for the activity (see text for details).

point to the last observation available on July 18 is $\sim 4.8 \times 10^{8} \mathrm{~kg}$. The maximum size of the particles ranges from $2-15 \mathrm{~cm}$, and the power-index of the size distribution is between -3.3 and -3.7 . The ejection velocity field is displayed in Fig. 7. The minimum velocity corresponds to the largest particles at any moment, which must always be greater than the escape velocity. To estimate the escape velocity, we adopted a nucleus radius $R_{\mathrm{n}}=0.46 \mathrm{~km}$ as reported in Fernández \& Sosa (2015), assuming a geometrical albedo of $\mathrm{p}_{v}=0.04$. Regarding its density, since we are exploring the potential main belt origin of this object, it may be asteroid-like, that is, $\sim 1000 \mathrm{~kg} \mathrm{~m}^{-3}$ (Carry 2012). However, its gas and dust production rates are more similar to a typical JFC with a bulk density of $\sim 550 \mathrm{~kg} \mathrm{~m}^{-3}$. Using an asteroidal density or a cometary density, the corresponding escape velocity from the nucleus at a distance of $20 R_{\mathrm{n}}$, where the gas drag vanishes, are 0.08 or $0.06 \mathrm{~m} \mathrm{~s}^{-1}$, respectively. We considered the comet-like and asteroid-like nature of $66 \mathrm{P}$ and adopted the mean value of $0.07 \mathrm{~m} \mathrm{~s}^{-1}$. As shown in Fig. 7, the maximum speed is $\sim 200 \mathrm{~m} \mathrm{~s}^{-1}$ at perihelion distance $(\sim 1.28 \mathrm{au})$ and achieved by the smallest particles in the model. The comparisons, found here, between the maximum speed and the results derived by other authors using Monte Carlo models or other dust models show that similar terminal velocities were derived for micron-sized particles ejected by other comets. (see e.g., Pozuelos et al. 2018; Moreno et al. 2017; Agarwal et al. 2007).

In Fig. 8, four images selected from the observational data set are compared with the corresponding synthetic images, which were generated by the best-fitting model. Due to a large number of time-dependent variables (e.g., $v_{0}(t), \delta(t), r_{\mathrm{dmax}}(t)$, and $\left.Q_{\text {dust }}(t)\right)$ in the model, it might be possible to find an alternative set of parameter values that could also fit the observational data. Further constraints on the model can be implemented by obtaining future observations that cover a significant orbital arc and include both pre- and post-perihelion, which was the case for comet 41P (see e.g., Pozuelos et al. 2018).

\section{Dynamical evolution}

Fernández \& Sosa (2015) defined a likely dynamical path to determine the degree of orbital stability for the near-Earth JFCs. 
Table 4. Log of observations used for dust modeling.

\begin{tabular}{ccccccc}
\hline \hline $\begin{array}{c}\text { Date } \\
\text { (UT) }\end{array}$ & $\begin{array}{c}\text { Days to } \\
\text { perihelion }\end{array}$ & $\begin{array}{c}\text { Resolution }^{(1)} \\
\left(\mathrm{km} \mathrm{pixel}^{-1}\right)\end{array}$ & $\begin{array}{c}\text { Dimension } \\
\left(\text { pixels }^{2}\right)\end{array}$ & $\begin{array}{c}\text { Phase } \\
\left.\text { angle }{ }^{\circ}\right)\end{array}$ & $\begin{array}{c}A\left(\theta=0^{\circ}\right) f \rho^{(3)} \\
(\mathrm{cm})\end{array}$ & $\begin{array}{c}A\left(\theta=0^{\circ}\right) f \rho^{\prime(4)} \\
(\mathrm{cm})\end{array}$ \\
\hline (a) 2018-05-23.4 & 2.4 & 832.7 & 40 & 51.3 & $86.8 \pm 4.3$ & $79.7 \pm 6.3$ \\
(b) 2018-06-14.4 & 24.4 & 847.6 & 50 & 49.3 & $62.5 \pm 3.1$ & $66.4 \pm 4.1$ \\
(c) 2018-06-28.4 & 38.4 & 856.0 & 30 & 46.7 & $37.8 \pm 1.8$ & $35.0 \pm 3.5$ \\
(d) 2018-07-19.4 & 59.4 & 856.0 & 40 & 40.5 & $29.8 \pm 1.5$ & $31.9 \pm 2.7$ \\
\hline
\end{tabular}

Notes. ${ }^{(1)}$ Resolution of the images in Fig. 8. ${ }^{(2)}$ Dimensions of the images in Fig. $8 .{ }^{(3)}$ Corresponding to the observations at $\rho=5000 \mathrm{~km}$. ${ }^{(4)}$ Corresponding to the synthetic images at $\rho=5000 \mathrm{~km}$.

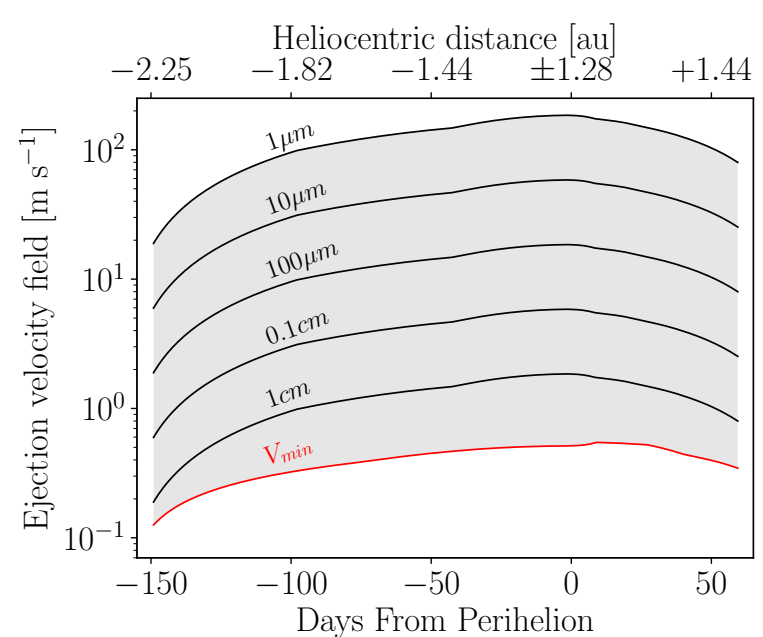

Fig. 7. Ejection-velocity field of best-fitting model, as function of heliocentric distance (upper $x$-axis) and day relative to perihelion (lower $x$-axis). The velocities of the $1 \mu \mathrm{m}, 10 \mu \mathrm{m}, 100 \mu \mathrm{m}, 0.1 \mathrm{~cm}$, and $1 \mathrm{~cm}$ sized particles are shown. The slowest velocity in the model is red-labeled as $V_{\min }$, which corresponds to the largest particles at any moment (from 2 to $15 \mathrm{~cm}$ ).

They find a strong correlation between the orbital stability and a set of critical parameters, which are the indices $f_{q}$ and $f_{a}$, the capture-time, $t_{\text {cap }}$, and the closest approach to Jupiter, $d_{\text {min }}$. In short, the $f_{q}$ index evaluates the time spent under the gravitational influence of Jupiter during the last $10^{4} \mathrm{yr}$, that is, the fraction of time during the last $10^{4} \mathrm{yr}$ that the comet (or any of its clones) moves along an orbit with $q>2.5 \mathrm{au}$, or reaches heliocentric distances $r_{\mathrm{h}}>100 \mathrm{au}$. The index $f_{a}$ refers to the time during which the comet (or any of its clones) orbits with $a>7.37 \mathrm{au}$, that is, the comet is no longer controlled by Jupiter. Additionally, the capture-time is the time in the past at which the mean perihelion, $\bar{q}(t)$, increased by one au with respect to the initial value at the discovery time. This parameter describes the time spent by a comet in Earth's vicinity. We refer the reader to Fernández \& Sosa (2015) for a full mathematical description of the indices $f_{q}, f_{a}$, and $t_{\text {cap }}$.

Unstable near-Earth JFCs have their $f_{q}$ and $f_{a} \gg 0$ and a small fraction of near-Earth JFCs with very stable orbits have nearly zero $f_{q}$ and $f_{a}$ as well as very large $t_{\text {cap }}\left(>5 \times 10^{4} \mathrm{yr}\right)$ and $\bar{d}_{\min }>0.3$ au (Fernández \& Sosa 2015). In this context, the comet $66 \mathrm{P}$ was found to have $f_{q}=f_{a}=0, t_{\text {cap }}>5 \times 10^{4} \mathrm{yr}$, and $\bar{d}_{\text {min }}>1.00$ au (2.87 Jupiter's hill radii). Thus, 66P is considered highly asteroidal and may have its origin in the asteroid belt.

After the last perihelion passage, the orbit of 66P has the best possible accuracy. We revisited and computed the parameters $f_{q}$, $f_{a}, t_{\text {cap, }}$ and $\bar{d}_{\text {min }}$ to verify the results of Fernández \& Sosa (2015) using the latest orbital elements. We used numerical integrations
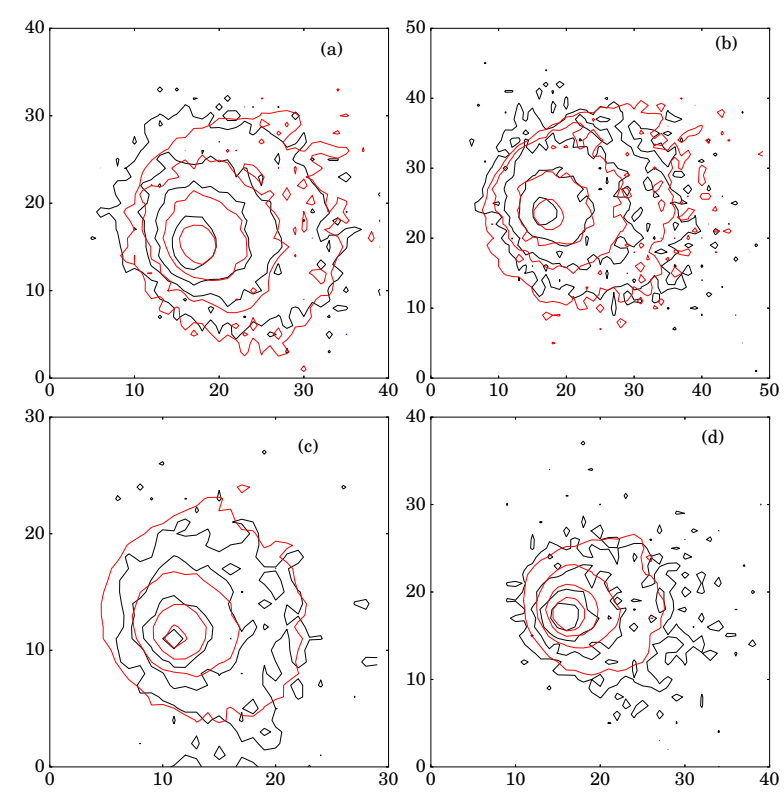

Fig. 8. Comparison of four observed (see Table 4) and modeled images. In all cases, the black contours correspond to observations and the red ones to the best-fitting model. The $y$ and $x$ axes are given in units of pixels. The plot is oriented so that north is up and east is to the left.

in the heliocentric frame, starting from January 1, 2019 and integrated backward up to $10^{5} \mathrm{yr}$. We adopted the numerical package MERCURY (Chambers 1999), with the Bulirsch-Stoer algorithm, which offers a high accuracy integration, but it is slow. To perform the statistical study, we generated 200 clones of the nominal orbit of $66 \mathrm{P}$, according to the associated $6 \times 6$ covariance matrix (Chernitsov et al. 1998).

We ran our simulations the second time to examine the influence of the non-gravitational forces; in this case, the covariance matrix includes two extra terms, which are the radial and transverse acceleration, respectively. Both sets of the orbital parameters and the covariance matrix of the orbit for $66 \mathrm{P}$ are published together in the NASA/JPL small-body browser. In order to ensure the highest accuracy possible, we integrated every clone independently, that is, we performed 200 simulations with one clone each. The initial time-step was set to five days and the computed orbital evolution was stored every year for each clone. The Sun, the eight planets, and Pluto were included in the simulation. Because of the weak activity of 66P, the simulations with and without non-gravitational forces yielded identical results. Hence, in the subsequent analysis we only show the results of the pure gravitational model. The dynamical history of $66 \mathrm{P}$ and its clones are shown in Fig. 9. We notice that the orbital evolution of $66 \mathrm{P}$ and of its clones are extremely compact during a period of $6.0 \times 10^{4} \mathrm{yr}$, with no significant divergence. During this 

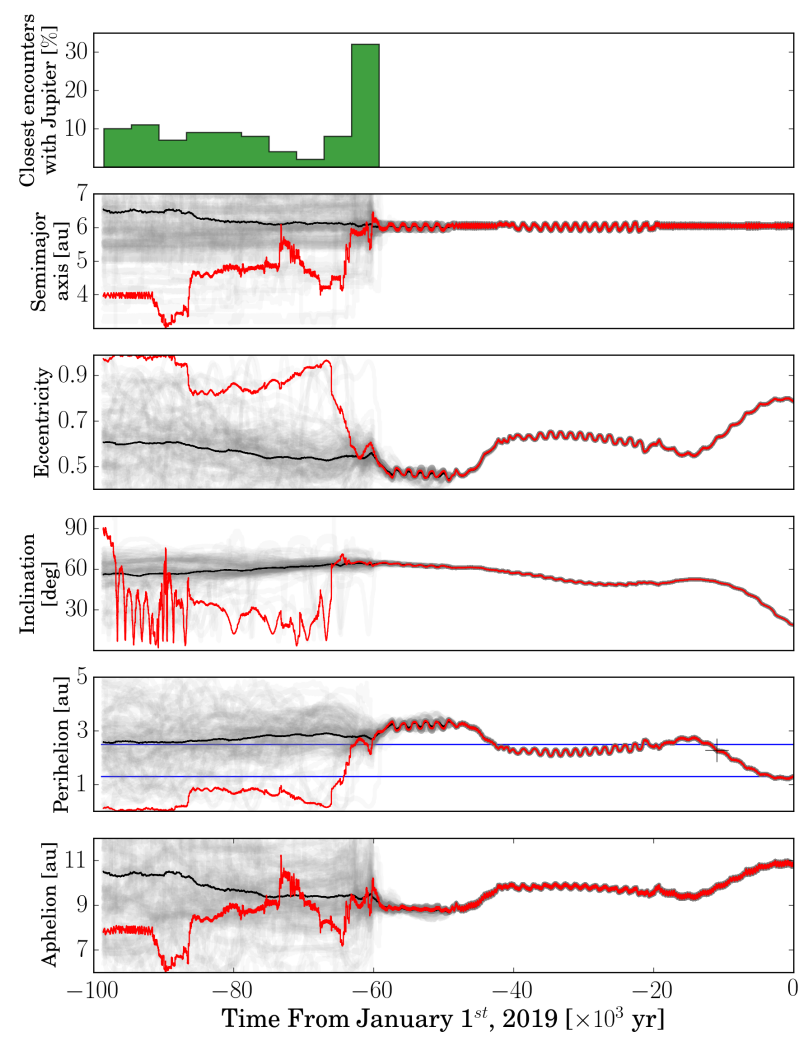

Fig. 9. Orbital evolution of $66 \mathrm{P}$ and its 200 clones for $10^{5} \mathrm{yr}$ backward in time from January 1, 2019. From the top to the bottom: the closest approaches with Jupiter, semi-major axis, eccentricity, inclination, perihelion, and aphelion distance. In all cases the red lines correspond to the evolution of the nominal comet 66P, the black lines are the mean values of the whole set of 200 clones plus the nominal comet, and the gray lines show the individual evolution for each clone. In the perihelion panel the black cross indicates the time of the capture, $t_{\text {cap }}$, and the two horizontal blue bars correspond to $q=1.3$ and $q=2.5$ au. The initial orbital elements were taken from the JPL Small-Body Data Browser.

period, the comet and the clones are safe from close encounters with Jupiter, with minimum distances greater than $1 \mathrm{au}$. At the time of approximately $-6.0 \times 10^{4} \mathrm{yr}$, the orbits begin to diverge and display chaotic behavior. This effect matches with the closest encounter with Jupiter of $\bar{d}_{\text {min }} \sim 0.2$ au (0.57 Jupiter's hill radii). About $30 \%$ of the clones have their closest encounter at that time, and the rest of them during the period -6 to $-10 \times 10^{4} \mathrm{yr}$. We find $f_{q}=0.0, f_{a}=0.0$, which are consistent with the values given in Fernández \& Sosa (2015). However, the capture time, $t_{\text {cap }} \sim 10^{4} \mathrm{yr}$, is about an order of magnitude shorter. According to the formal definition presented in Fernández \& Sosa (2015), $66 \mathrm{P}$ can no longer be categorized as highly asteroidal because of the shorter $t_{\text {cap }}$. Instead, 66P is only moderately asteroidal and therefore cannot be considered as a NEMBC candidate.

On the other hand, our simulations show that $q$ oscillates between 1.3 and $2.5 \mathrm{au}$ for $\sim 4.0 \times 10^{4} \mathrm{yr}$. This pattern is similar to the behavior of most near-Earth asteroids, whose orbits are stable for $10^{4} \mathrm{yr}$ or longer, with perihelion distances confined to $q<2.5 \mathrm{au}$ and semi-major axes to $a<7.37 \mathrm{au}$. Many of these asteroids are trapped in mean motion resonances (MMRs) with Jupiter (Fernández et al. 2014). In addition, we noticed that the semimajor axis of 66P oscillates around a quasi-constant value of $6 \mathrm{au}$, so we calculated the strength for all possible resonances located near this value following Gallardo (2006). We find that $66 \mathrm{P}$ is trapped in 4:5 MMR with Jupiter from 0 to $-60 \times 10^{3} \mathrm{yr}$, with different states of the critical angle, $\sigma_{\mathrm{c}}$, as shown in Fig. 10.

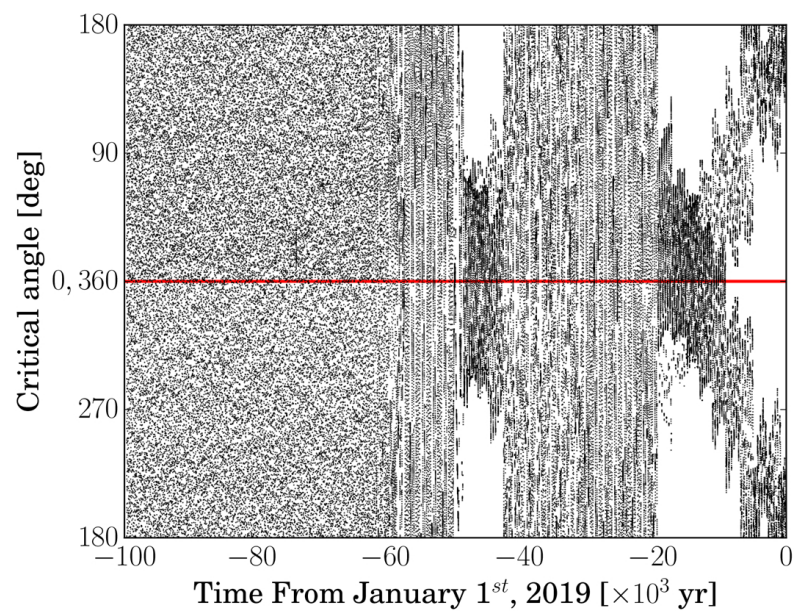

Fig. 10. Evolution of critical angle of 66P defined as $\sigma_{\mathrm{c}}=(p+q) \lambda_{\mathrm{J}}-$ $p \lambda_{c}-q \bar{\omega}$, where $|p+q|:|p|$ corresponds to MMR with Jupiter, $\lambda_{\mathrm{J}}$ is mean longitude of Jupiter, $\lambda_{c}$ mean longitude of $66 \mathrm{P}$, and $\bar{\omega}$ is longitude of perihelion.

During the time that $66 \mathrm{P}$ is trapped in the MMR, the evolution of $\sigma_{\mathrm{c}}$ is complex, which alternates between periods of libration and periods of circulation. From 0 to $-5000 \mathrm{yr}, \sigma_{\mathrm{c}}$ librates around $180^{\circ}$, with a large semi-amplitude of $90^{\circ}$. From -5000 to $-10 \times 10^{3} \mathrm{yr}$ it gently evolves toward $0^{\circ}$, where it remains until $-20 \times 10^{3} \mathrm{yr}$. From $-20 \times 10^{3}$ to $-45 \times 10^{3} \mathrm{yr} \sigma_{\mathrm{c}}$ circulates, and back again to librate around $0^{\circ}$ from $-45 \times 10^{3}$ to $-50 \times 10^{3} \mathrm{yr}$. Finally, from $-50 \times 10^{3}$ to $-60 \times 10^{3} \mathrm{yr}$ it circulates again, before showing chaotic behavior, presumably due to the close encounter with Jupiter at this time. It is interesting to note that during the period that $\sigma_{\mathrm{c}}$ circulates, there is a coupling of the orbital parameters as shown in Fig. 9. Although $i$ shows very little cyclic variations, it may be coupled with $q$, indicating the action of the Kozai mechanism, where the parameter $H=\sqrt{1-e^{2}} \cos (i)$ remains constant. We computed the evolution of $H$, and we obtained a non-constant value that varied from 0.60 to 0.35 during the time that $66 \mathrm{P}$ was trapped in the MMR from 0 to $-60 \times 10^{3} \mathrm{yr}$. As such, we conclude that the Kozai mechanism is not responsible for the coupling of the orbital parameters.

\section{Discussion}

Based on our UVES observations of 66P, we derived an $\mathrm{NH}_{3}$ OPR of $1.08 \pm 0.06$ and a spin temperature of $34 \mathrm{~K}$, which are consistent with the values observed in others JFCs (Shinnaka et al. 2016). Traditionally, the OPRs exhibited by gaseous species observed in cometary comae have been used to constrain the formation temperature of comets, assuming that OPRs have been unchanged in cometary nuclei since the formation of the molecules about 4.6 billion years ago. However, there are several problems with this view. As shown in Fig. 4, most comets have $\mathrm{NH}_{3}$ OPRs between 1.1 and 1.2 , corresponding to a spin temperature of $\sim 30 \mathrm{~K}$. This temperature is significantly higher than $10 \mathrm{~K}$ as suggested by the theoretical studies on ${ }^{15} \mathrm{~N}$-fractionation in ammonia (Shinnaka et al. 2016). Recently, laboratory studies have found that OPR of $\mathrm{H}_{2} \mathrm{O}$ can be modified and re-equilibrated via interactions with other molecules or with the solid matrix (Hama et al. 2016, and references therein). The new findings of the laboratory studies suggest that it is incorrect to assume the OPRs of cometary species are primordial and the OPRs can not be used to derive the formation temperature of certain molecules, such as $T_{\text {spin }}$. More detailed discussions on OPRs are present in Shinnaka et al. (2016). Similar to water, the OPR of ammonia can 
A\&A 631, A168 (2019)

Table 5. Upper limits of $\mathrm{CN}$ and $\mathrm{H}_{2} \mathrm{O}$ production rates and dust production rates of MBCs (Snodgrass et al. 2017a) compared to 66P.

\begin{tabular}{|c|c|c|c|c|c|c|c|c|}
\hline Objects & $\begin{array}{c}\mathrm{Q}\left(\mathrm{H}_{2} \mathrm{O}\right)_{s}^{(a)} \\
\left(10^{26} \mathrm{molec} \mathrm{s}^{-1}\right)\end{array}$ & $\begin{array}{c}\mathrm{Q}(\mathrm{CN})_{s}^{(a)} \\
\left(10^{24} \mathrm{molec} \mathrm{s}^{-1}\right)\end{array}$ & $\begin{array}{c}\frac{\mathrm{d} M}{\mathrm{~d} t}{ }_{s}^{(b)} \\
\left(\mathrm{kg} \mathrm{s}^{-1}\right) \\
\end{array}$ & $\begin{array}{c}\mathrm{r}_{h} \\
(\mathrm{au}) \\
\end{array}$ & $\begin{array}{c}\mathrm{Q}\left(\mathrm{H}_{2} \mathrm{O}\right) \\
\left(10^{26} \text { molec s}^{-1}\right)\end{array}$ & $\begin{array}{c}\mathrm{Q}(\mathrm{CN}) \\
\left(10^{24} \mathrm{molec} \mathrm{s}^{-1}\right)\end{array}$ & $\begin{array}{c}\frac{\mathrm{d} M}{\mathrm{~d} t} \\
\left(\mathrm{~kg} \mathrm{~s}^{-1}\right)\end{array}$ & References \\
\hline $133 \mathrm{P}$ & 0.1 & 0.1 & 7.2 & 2.64 & 0.02 & 0.01 & 1.4 & Licandro et al. (2011) \\
\hline $176 \mathrm{P}$ & 2.6 & - & 0.5 & 2.58 & 0.40 & - & 0.1 & de Val-Borro et al. (2012) \\
\hline $259 \mathrm{P}$ & 1.3 & 0.4 & - & 1.86 & 0.50 & 0.14 & - & Jewitt et al. (2009) \\
\hline $288 \mathrm{P}$ & 6.1 & 2.6 & 2.3 & 2.52 & 1.00 & 0.42 & 0.5 & Hsieh et al. (2012a) \\
\hline $313 P$ & 3.2 & 1.6 & 1.7 & 2.41 & 0.60 & 0.18 & 0.4 & Jewitt et al. (2015) \\
\hline $324 \mathrm{P}$ & 7.1 & 1.9 & 1.1 & 2.66 & 1.00 & 0.30 & 0.2 & Hsieh et al. (2012b) \\
\hline $358 \mathrm{P}$ & 2.7 & 0.8 & 4.3 & 2.42 & 0.50 & 0.15 & 1.0 & Hsieh et al. (2013) \\
\hline P/2013 R3 & 1.9 & 1.2 & $<3.5$ & 2.23 & 0.43 & 0.12 & $<1.0^{(c)}$ & Jewitt et al. (2014a) \\
\hline $66 \mathrm{P}$ & 27.1 & 7.5 & 55.0 & 1.29 & 27.1 & 7.5 & 55.0 & This work \\
\hline
\end{tabular}

Notes. ${ }^{(a)}$ Scaled water and $\mathrm{CN}$ production rates to $r_{\mathrm{h}}=1.29$ au, using $\mathrm{Q}($ gas $) \propto r_{\mathrm{h}}^{-2.7}$ given by A'Hearn et al. (1995). ${ }^{(b)}$ Scaled dust production rates to $r_{\mathrm{h}}=1.29 \mathrm{au}$, using the proxy relationship: $\frac{\mathrm{d} M}{\mathrm{~d} t} \propto r_{\mathrm{h}}^{-2.3}$, given by A'Hearn et al. (1995). ${ }^{(c)}$ The empirical limit to the mass loss, from Jewitt et al. (2017).

be modified in the coma by electron recombination, we, therefore, can no longer use the derived $\mathrm{NH}_{3}$ OPR to constrain the nucleus formation temperature and, in turn, to verify the origin of $66 \mathrm{P}$.

Although $66 \mathrm{P}$ is a weakly active comet, thanks to its close distance to the Earth at the time of observations, several gas species were detected both with X-shooter/VLT and TRAPPIST. The relative abundances, such as the $\mathrm{Q}(\mathrm{CN}) / \mathrm{Q}(\mathrm{OH})$ ratio versus the $\mathrm{Q}\left(\mathrm{C}_{2}\right) / \mathrm{Q}(\mathrm{OH})$ ratio of $66 \mathrm{P}$ as well as those of JFCs and Oort cloud comets are shown in Fig. 11. The $\mathrm{Q}(\mathrm{CN}) / \mathrm{Q}(\mathrm{OH})$ and $\mathrm{Q}\left(\mathrm{C}_{2}\right) / \mathrm{Q}(\mathrm{OH})$ of $66 \mathrm{P}$ are slightly lower than other JFCs; however, its relative abundance ratios are within the normal values of over 100 typical comets studied in (Schleicher 2008; A'Hearn et al. 1995). We note that 66P was observed only within a narrow time window. Nevertheless, the TRAPPIST observations of 66P were made at typical heliocentric distances for other JFC observations, so it is reasonable to compare our results with other JFC measurements.

A'Hearn et al. (1995) noted that for a typical comet all gaseous species vary at a similar rate with the heliocentric distance, which can be described as $\mathrm{Q}($ gas $) \propto r_{\mathrm{h}}^{-2.7}$. Given that the activity of MBCs is likely to be driven by water ice sublimation, we scaled the upper limits of water and $\mathrm{CN}$ production rates of the known MBCs to $r_{\mathrm{h}}=1.29 \mathrm{au}$, where most observations were made for 66P. The scaled values as well as the original upper limits are listed in Table 5. $\mathrm{Q}\left(\mathrm{H}_{2} \mathrm{O}\right)$ of $66 \mathrm{P}$ is about an order of magnitude higher than those of known MBCs. One caveat of this comparison is that most upper limits of $\mathrm{Q}\left(\mathrm{H}_{2} \mathrm{O}\right)$ of $\mathrm{MBCs}$ are scaled from $\mathrm{Q}(\mathrm{CN})$, assuming a cometary ratio $\mathrm{Q}(\mathrm{CN}) / \mathrm{Q}\left(\mathrm{H}_{2} \mathrm{O}\right) \sim 0.001$. MBCs are closer to the Sun than typical comets, therefore $\mathrm{CN}$ could be depleted in MBCs (Prialnik \& Rosenberg 2009). However, for $176 \mathrm{P}$ and 358P, the $\mathrm{Q}\left(\mathrm{H}_{2} \mathrm{O}\right)$ rates were derived by observing the $\mathrm{H}_{2} \mathrm{O}$ and $\mathrm{OH}$ lines directly (de Val-Borro et al. 2012; O'Rourke et al. 2013), which are comparable to the limits derived using $\mathrm{Q}(\mathrm{CN})$.

The optical colors and spectra of known MBCs are mostly similar to those of the C-class asteroids (DeMeo et al. 2009). Only one MBC, 358P/PANSTARRS, has been observed in the NIR and the comet appears redder in the NIR as shown in Fig. 2. However, 358P was very faint at the time of the observations and its NIR spectrum is rather noisy and heavily affected by the telluric absorptions. Our X-shooter observations show that the D-type like spectrum of 66P is significantly different from those of MBCs and is more similar to the spectra of active JFCs,

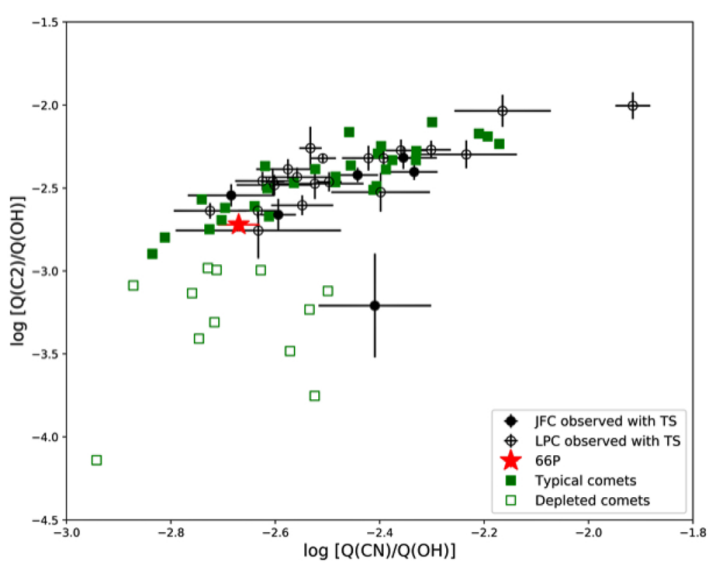

Fig. 11. Logarithm of ratio of $\mathrm{C}_{2}$ to $\mathrm{OH}$ production rates as function of logarithm of ratio of $\mathrm{CN}$ to $\mathrm{OH}$ for 66P (red star) compared to JFCs (filled circles) and long-period comets (crosses) observed with TRAPPIST between 2010 and 2016 (Opitom 2016), and also with typical (green filled squares) and carbon-chain depleted (green opened squares) comets given in A'Hearn et al. (1995).

such as $6 \mathrm{P} / \mathrm{d}$ 'Arrest. We would like to point out that the MBC spectra as well as the spectrum of $66 \mathrm{P}$ were obtained when these objects were active. Therefore, the spectra do not necessarily reflect the intrinsic composition of the nuclei because the nuclei were buried inside the dust comae along the light-of-sight. Besides composition, the spectral slope can also be affected by the size distribution of the dust coma. Although we can not exclusively conclude that the intrinsic composition of 66P differs from those of the MBCs, our observations suggest that the composition and/or the particle size distribution of the coma of 66P are more similar to those of the JFCs than the MBCs.

Moreover, our dust models find remarkable differences between 66P and the MBCs, the latter in general show much lower values for both dust production rates $\left(0.2-1.4 \mathrm{~kg} \mathrm{~s}^{-1}\right)$ and ejection velocities $\left(0.5-2.0 \mathrm{~m} \mathrm{~s}^{-1}\right.$ for particles of $\left.100 \mu \mathrm{m}\right)$ (see e.g., Hsieh et al. 2009; Jewitt et al. 2014b; Pozuelos et al. 2015; Agarwal et al. 2016). In comparison, the JFCs have typical dust productions rates of $40-250 \mathrm{~kg} \mathrm{~s}^{-1}$ and ejection velocities for particles of $100 \mu \mathrm{m}$ ranging from 5 to $10 \mathrm{~m} \mathrm{~s}^{-1}$ (see e.g., Pozuelos et al. 2014b,a; Moreno et al. 2017). The previous statistical study of 85 comets found the average variation of the dust production rate can be expressed as $A(\theta) f \rho \propto r_{\mathrm{h}}^{-2.3}$ (A'Hearn et al. 1995). 
When scaling to $r_{\mathrm{h}}=1.29 \mathrm{au}$, the dust production rates of MBCs would be in the range of $1-10 \mathrm{~kg} \mathrm{~s}^{-1}$, as listed in the Table 5, which are at least one order of magnitude smaller than the dust production rate of $66 \mathrm{P}$.

Using the latest orbital elements, our dynamical simulation confirmed that the orbit of 66P is stable with $f_{q}$ and $f_{a}$ equal to 0 . However, our model found a much shorter $t_{\text {cap }}$. Therefore, 66P is no longer highly asteroidal and can not be considered as an NEMBC candidate. The discrepancy between our results and those of Fernández \& Sosa (2015) may either be caused by the quality of the orbital elements or by the number of clones and how they are generated. Fernández \& Sosa (2015) used a simple Gaussian distribution with a standard deviation given by the nominal uncertainties to generate 50 clones. In contrast, we used a more robust method based on the covariance matrix and we generated 200 clones in this study. On the other hand, a detailed exploration of its dynamical evolution shows that 66P does share some features with near-Earth asteroids.

For other NEMBC candidates identified in Fernández \& Sosa (2015), Fernández et al. (2017) studied the anomalous comet 249P/Linear and found that its activity lasts only $\sim 20$ days around perihelion with a peak mass loss rate of $145 \mathrm{~kg} \mathrm{~s}^{-1}$ that is much higher than the dust production rates of MBCs. The spectrum of 249P was found to be similar to B-type asteroids, a trait that is also shared with some MBCs. Although the dynamical simulations show some similarities for 249P and 66P, the physical properties of these two objects are very different.

\section{Summary and conclusion}

We performed detailed physical studies of the near-Earth JFC: $66 \mathrm{P}$, our main results are:

Firstly, based on the UVES/VLT observations, a suit of $\mathrm{NH}_{2}$ lines were detected. The OPR of $\mathrm{NH}_{3}$ using the highdispersion spectrum of $\mathrm{NH}_{2}$ was found to be $1.08 \pm 0.06$ and its $T_{\text {spin }}=34 \mathrm{~K}_{-5}^{+12}$, which are comparable to the values of other normal JFCs.

Secondly, based on the X-shooter/VLT and the TRAPPIST observations, common cometary gaseous species such as, $\mathrm{OH}$, $\mathrm{CN}, \mathrm{C}_{2}$, and $\mathrm{C}_{3}$ were detected. The relative abundances of $66 \mathrm{P}$ is consistent with those of typical JFCs. The reflectance spectrum of 66P closely resembles the mean spectrum of the D-type asteroids, which is much redder than the spectra of the known MBCs.

Thirdly, dust models using the TRAPPIST observations obtained the peak mass loss rate of $55 \mathrm{~kg} \mathrm{~s}^{-1}$ that is about an order of magnitude larger than the mass loss rates of the MBCs when scaled to the same heliocentric distance.

Lastly, dynamical simulations using the latest orbital elements of $66 \mathrm{P}$ found that it is no longer highly asteroidal but moderately asteroidal due to the shorter capture time.

Considering all the available observations as well as the results of the dust model and the dynamical model, we conclude that $66 \mathrm{P}$ is much more similar to typical JFCs than MBCs and, therefore, it is unlikely to have originated from the asteroid main belt and it is not related to MBCs.

Acknowledgements. We would like to thank the anonymous referee for his/her careful review and constructive suggestions. Based on observations collected at the European Organisation for Astronomical Research in the Southern Hemisphere under ESO program 2101.C-5033(A) and (B). TRAPPIST-South is funded by the Belgian Fund for Scientific Research (Fond National de la Recherche Scientifique, FNRS) under the grant FRFC 2.5.594.09.F. E.J. and D.H. are Belgian FNRS Senior Research Associates. Simulations in this paper made use of the REBOUND code which can be downloaded freely at http://github. com/hannorein/rebound.

\section{References}

Agarwal, J., Müller, M., \& Grün, E. 2007, Space Sci. Rev., 128, 79 Agarwal, J., Jewitt, D., Weaver, H., Mutchler, M., \& Larson, S. 2016, AJ, 151, 12 A'Hearn, M. F., Schleicher, D. G., Millis, R. L., Feldman, P. D., \& Thompson, D. T. 1984, AJ, 89, 579

A'Hearn, M. F., Millis, R. C., Schleicher, D. O., Osip, D. J., \& Birch, P. V. 1995, Icarus, 118, 223

Ballester, P., Modigliani, A., Boitquin, O., et al. 2000, The Messenger, 101, 31 Bentley, M. S., Schmied, R., Mannel, T., et al. 2016, Nature, 537, 73

Carry, B. 2012, Planet. Space Sci., 73, 98

Chambers, J. E. 1999, MNRAS, 304, 793

Chernitsov, A. M., Baturin, A. P., \& Tamarov, V. A. 1998, Sol. Syst. Res., 32, 405

Cochran, A. L., \& Schleicher, D. G. 1993, Icarus, 105, 235

de Val-Borro, M., Rezac, L., Hartogh, P., et al. 2012, A\&A, 546, L4

Della Corte, V., Rotundi, A., Fulle, M., et al. 2016, MNRAS, 462, S210

DeMeo, F. E., Binzel, R. P., Slivan, S. M., \& Bus, S. J. 2009, Icarus, 202, 160

Farnham, T. L., Schleicher, D. G., \& A'Hearn, M. F. 2000, Icarus, 147, 180

Fernández, J. A., \& Sosa, A. 2015, Planet. Space Sci., 118, 14

Fernández, J. A., Sosa, A., Gallardo, T., \& Gutiérrez, J. N. 2014, Icarus, 238, 1

Fernández, J. A., Licandro, J., Moreno, F., et al. 2017, Icarus, 295, 34

Fornasier, S., Hasselmann, P. H., Barucci, M. A., et al. 2015, A\&A, 583, A30

Freudling, W., Romaniello, M., Bramich, D. M., et al. 2013, A\&A, 559, A96

Fulle, M., Della Corte, V., Rotundi, A., et al. 2016a, MNRAS, 462, S132

Fulle, M., Marzari, F., Della Corte, V., et al. 2016b, ApJ, 821, 19

Gallardo, T. 2006, Icarus, 184, 29

Hama, T., Kouchi, A., \& Watanabe, N. 2016, Science, 351, 65

Haser, L. 1957, Bull. Soc. Roy. Sci. Liège, 43, 740

Hsieh, H. H., \& Haghighipour, N. 2016, Icarus, 277, 19

Hsieh, H. H., \& Jewitt, D. 2006, Science, 312, 561

Hsieh, H. H., Jewitt, D., \& Fernández, Y. R. 2009, ApJ, 694, L111

Hsieh, H. H., Yang, B., \& Haghighipour, N. 2011, ApJ, 744, 9

Hsieh, H. H., Yang, B., Haghighipour, N., et al. 2012a, ApJ, 748, L15

Hsieh, H. H., Yang, B., Haghighipour, N., et al. 2012b, AJ, 143, 104

Hsieh, H. H., Kaluna, H. M., Novaković, B., et al. 2013, ApJ, 771, L1

Jehin, E., Gillon, M., Queloz, D., et al. 2011, The Messenger, 145, 2

Jewitt, D., Yang, B., \& Haghighipour, N. 2009, AJ, 137, 4313

Jewitt, D., Agarwal, J., Li, J., et al. 2014a, ApJ, 784, L8

Jewitt, D., Ishiguro, M., Weaver, H., et al. 2014b, AJ, 147, 117

Jewitt, D., Agarwal, J., Peixinho, N., et al. 2015, AJ, 149, 81

Jewitt, D., Agarwal, J., Li, J., et al. 2017, AJ, 153, 223

Kawakita, H., Watanabe, J., Ando, H., et al. 2001, Science, 294, 1089

Licandro, J., Campins, H., Tozzi, G., et al. 2011, A\&A, 532, A65

Licandro, J., Moreno, F., de León, J., et al. 2013, A\&A, 550, A17

Manfroid, J., Jehin, E., Hutsemékers, D., et al. 2009, A\&A, 503, 613

Mannel, T., Bentley, M. S., Schmied, R., et al. 2016, MNRAS, 462, S304

Modigliani, A., Goldoni, P., Royer, F., et al. 2010, SPIE Conf. Ser., 7737, 773728

Monet, D. G., Levine, S. E., Canzian, B., et al. 2003, AJ, 125, 984

Moreno, F., Pozuelos, F., Aceituno, F., et al. 2012, ApJ, 752, 136

Moreno, F., Pozuelos, F., Aceituno, F., et al. 2014, ApJ, 791, 118

Moreno, F., Licandro, J., Cabrera-Lavers, A., \& Pozuelos, F. J. 2016a, ApJ, 826, 137

Moreno, F., Licandro, J., Cabrera-Lavers, A., \& Pozuelos, F. J. 2016b, ApJ, 826, L22

Moreno, F., Muñoz, O., Gutiérrez, P. J., et al. 2017, MNRAS, 469, S186

Moulane, Y., Jehin, E., Opitom, C., et al. 2018, A\&A, 619, A156

Opitom, C. 2016, $\mathrm{PhD}$ Thesis, University of Liège, Belgium

Opitom, C., Jehin, E., Manfroid, J., et al. 2015a, A\&A, 574, A38

Opitom, C., Jehin, E., Manfroid, J., et al. 2015b, A\&A, 584, A121

Opitom, C., Guilbert-Lepoutre, A., Jehin, E., et al. 2016, A\&A, 589, A8

O'Rourke, L., Snodgrass, C., de Val-Borro, M., et al. 2013, ApJ, 774, L13

Ott, T., Drolshagen, E., Koschny, D., et al. 2017, MNRAS, 469, S276

Pozuelos, F. J., Moreno, F., Aceituno, F., et al. 2014a, A\&A, 571, A64

Pozuelos, F. J., Moreno, F., Aceituno, F., et al. 2014b, A\&A, 568, A3

Pozuelos, F. J., Cabrera-Lavers, A., Licandro, J., \& Moreno, F. 2015, ApJ, 806, 102

Pozuelos, F. J., Jehin, E., Moulane, Y., et al. 2018, A\&A, 615, A154

Prialnik, D., \& Rosenberg, E. D. 2009, MNRAS, 399, L79

Riedler, W., Torkar, K., Jeszenszky, H., et al. 2007, Space Sci. Rev., 128, 869

Rotundi, A., Sierks, H., Della Corte, V., et al. 2015, Science, 347, aaa3905

Schleicher, D. G. 2008, AJ, 136, 2204

Sekanina, Z. 1981, Ann. Rev. Earth Planet. Sci., 9, 113

Shinnaka, Y., Kawakita, H., Jehin, E., et al. 2016, MNRAS, 462, S195

Snodgrass, C., Agarwal, J., Combi, M., et al. 2017a, A\&ARv, 25, 5

Snodgrass, C., Yang, B., \& Fitzsimmons, A. 2017b, A\&A, 605, A56

Whipple, F. L. 1951, ApJ, 113, 464

Yang, B. 2009, PhD Thesis, University of Hawai'i at Manoa 